\title{
MIXR: A Standard Architecture for Medical Image Analysis in Augmented and Mixed Reality
}

\author{
Benjamin Allison, Xujiong Ye, Faraz Janan \\ School of Computer Science \\ University of Lincoln \\ Lincoln, Country \\ ballison@lincoln.ac.uk
}

\begin{abstract}
Medical image analysis is evolving into a new dimension: where it will combine the power of $\mathrm{AI}$ and machine learning with real-time, real-space displays, namely Virtual Reality (VR), Augmented Reality (AR) and Mixed Reality (MR) - known collectively as Extended Reality (XR). These devices, typically available as head-mounted displays, are enabling the move towards the complete transformation of how medical data is viewed, processed and analysed in clinical practice. There have been recent attempts on how XR gadgets can help in surgical planning and training of medics. However, the radiological front from a detection, diagnostics and prognosis remains unexplored. In this paper we propose a standard framework or architecture called Medical Imaging in Extended Reality (MIXR) for building medical image analysis applications in XR. MIXR consists of several components used in literature; however, tied together for reconstructing volume data in $3 \mathrm{D}$ space. Our focus here is on the reconstruction mechanism for $\mathrm{CT}$ and MRI data in XR; nevertheless, the framework we propose has applications beyond these modalities.
\end{abstract}

Index Terms-Extended Reality, Mixed Reality, Augmented Reality, Medical Image Analysis

\section{INTRODUCTION}

There are very few proof-of-concept attempts within mixed reality (MR) that are largely driven to train or prepare clinicians for surgical interventions. These methods combine augmented reality (AR) to medical image representations; nevertheless, the medical image analysis front remains unexploited that has potential to entirely transform clinical radiology. While most attempts rely on hard-built models of human organs or synthetic data used for training clinicians, none of those known to us can deal with direct real-time patient data. With cancer imagery in mind, we propose a solution to visualise magnetic resonance imaging (MRI) and computed tomography (CT), which essentially is a $3 \mathrm{D}$ acquisition process, in an actual 3D paradigm. The solution aims to give a better approach to how visualisation and volume exploration is provided to clinicians. The goal of the research is to develop and combine science behind various aspects of extended reality (XR) to yield a solution for reconstructing diagnostic data. The solution can then be ported and tested on XR hardware platforms.

To expand on XR, virtual reality (VR) devices such as Sony, Oculus, and HTC Immerse uses a fully artificial digital environment; whereas AR technologies such as Google Glass work by overlaying virtual objects on the real-world environment. Mixed reality (MR) technologies such as the Microsoft HoloLens take the concept of AR further with not just overlaying but anchoring virtual objects to the real world. AR and MR research has displayed potential within medical image analysis in to surgical aids [1] as well within medical training [6].

XR has already had significant interest within the field of medical image analysis. Showing a range of potential use cases; however, a large sector of research in XR within medicine is focused on augmentation of surgery. Augmenting surgical procedures with $\mathrm{AR}$ has been proven to provide promising results [7], [8]. While AR and MR devices are more common, medical image analysis research into MR devices in radiology [9], [10] are sparse and yet to be proven. Their research highlights the applicability of MR devices in radiology as well as the lack of development in the field. These applications include using MR devices for medical autopsy, 3D visualisation of specimens, telepathology and specimen co registration. They highlight how the HoloLens introduces new novel approaches that could improve upon radiological practice. The ability to allow telepathology, interacting in real-time with the data with other radiologists provides a practical aspect other technologies do not provide. XR allows for virtual workstations and new ways of exploiting dynamic ways to manipulate volumetric data. The medium can provide a transformative solution that is safer [10] and provides a lower cognitive burden on the users [11]. It has diversity in how it can be applied within radiology and there is great opportunity for additional research in diagnosis.

\section{Extended Reality in Medical Image Analysis}

We have seen limited examples of fully fledged XR systems utilising the available functionality XR device offer. We have seen systems such as [18] that handle user input through gestures as controls to interact with volumetric data. These systems have been experimented with and found that a 'touchless' environment is easy to operate and creates a positive user experience regarding observation and interaction. With the basic feature extraction, they were able to create a system that was easier to use than that of traditional inputs. However, the system was not utilising XR, gesture recognition was done through a "Leap Motion device" and users had the 3D volume visualised still on a 2D screen, giving a disconnect between 


\begin{tabular}{|c|c|c|c|c|c|c|}
\hline \multicolumn{2}{|c|}{ Ledical Image Analysis } \\
\hline $\begin{array}{c}\text { Imaging } \\
\text { Modality }\end{array}$ & Liver & Lung & Breast & Brain & Pathology \\
\hline MRI & L. Shi et al [1] & P. Bettati et al [2] & S. L. Perkins et al [3] & A. Alfonso-Garcia et a [4] & $\begin{array}{c}\text { F. Chessa et al [5] } \\
\text { S. L. Perkins et al [3] } \\
\text { L. Shi et al [1] } \\
\text { V. Ferrari et al [13] }\end{array}$ \\
\hline CT & $\begin{array}{c}\text { L. Trestioreanu } \\
\text { et al [14] }\end{array}$ & F. King et al [15] & D. Douglas et al [12] & M. Hanna et al [9] & $\begin{array}{c}\text { J. Sutherland et al [10] } \\
\text { F. Cutolo et al [17] }\end{array}$ & $\begin{array}{c}\text { V. Ferrari et al [13] } \\
\text { X. Wu et al [16] } \\
\text { F. Cutolo et al [17] }\end{array}$ \\
\hline
\end{tabular}

TABLE I: Key works in extended reality applied to medical imaging

the user and their spatial understanding of the volume. There are limited examples of systems utilising XR. For instance, [9] showed the HoloLens, a mixed reality device being used for co-registering radiology, gross anatomy, and histopathology. They tested the system, finding patholgists, clinical assistants and trainees all found the system easy to manipulate whilst performing a variety of clinical and nonclinical use cases. They highlight the shortcomings of the research however, they identify the HoloLens has much wider potential. Unfortunately [9] failed to harness the potential of XR for radiology. With voice commands, documentation, annotation, video recording such a system can be taken much further in pathology. A system such as proposed by [10], utilises both the interactivity elements used by [18] but with 3D volumetric visualisation of the volume as discussed by [10]. We have seen sparse examples of framework for XR systems for medical image analysis. Listed in table $\mathrm{I}$ is a summation of frameworks for medical image analysis on MRI and CT data.

In this paper we develop a standardised architecture that we call MIXR, for XR applications in medical image analysis. We consider MIXR reproducible, founded on components wellgrounded in literature, easy to implement and thus comparable. This paper serves as a tutorial for readers who want to familiarise themselves with XR medical image analysis, as well as would act as a standard benchmark for architectures in future - a standard that does not exist to this end. In the following sections, first we will discuss the visualisation process of CT and MRI in XR. Then explain the tools and methods used in XR visualisation, which are also the fundamental components of MIXR. Further, we will devolve into the system overview - which is a use-case of functionalities for a medical image analysis XR system. Here, as an example we have implemented a basic computer aided diagnostic system for breast cancer to demonstrate a few core functionalities; however the architecture is adoptable for any other type of cancer imaging application - and further extendable to include more features, such as AI and beyond. Finally we discuss the future directions for XR in medical imaging.

\section{RADiOLOGICAL DATA VISUALISATION}

\section{A. CT and MRI Reconstruction}

This section discusses the methods and tools used in the MIXR architecture. Please note that unlike the conventional meaning of volume reconstruction, here we refer to the word "reconstructed" as a data volume rendered for visualisation in XR space. The volume data in its simplest definition is a

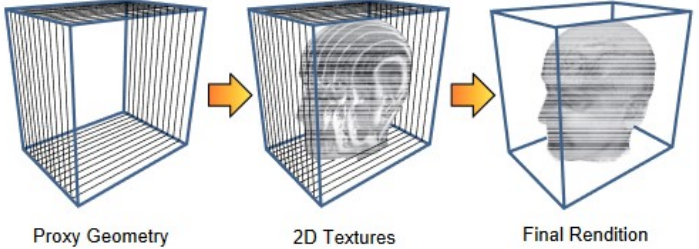

Fig. 1: [19] Object-aligned slices used as proxy geometry with $2 \mathrm{D}$ texture mapping

quadruple $(\mathrm{x}, \mathrm{y}, \mathrm{z}, \mathrm{w})$ where $\mathrm{x}, \mathrm{y}, \mathrm{z}$ is a scalar describing the $3 \mathrm{D}$ position of the volume data and $\mathrm{w}$ is the data value(s), this is often referred to as a voxel. To render the volume from this data it must first be reconstructed in a voxel-based volume as typically MRI and CT sources provide as a stack of $2 \mathrm{D}$ images. This reconstruction is performed with a similar method illustrated in figure 1 . The proxy geometry refers to a cube mesh which is created within Unity [20] to hold the volumetric material. The volume data can be reconstructed into a $3 \mathrm{D}$ object space by taking a series of $2 \mathrm{D}$ textures and stacking them together to produce a voxel. The material itself unlike a traditional object with a mesh and material cannot be visualised without the aid of volumetric rendering technique to achieve the final rendition.

\section{B. Volumetric Rendering}

Volume rendering algorithms play an integral part within the medical field to visualise 3D Data. Volume rendering is a technique for visualizing $3 \mathrm{D}$ data by computing $2 \mathrm{D}$ projections of a 3D volume. These algorithms can be divided into three algorithmic categories demonstrated in table II according to the order of traversal that the algorithm takes through the volume. The first, image order algorithms [21] which we will take a closer look at, iterate over the pixels in an image rather than the scene. Object order algorithms [22], [23] iterate over elements within a scene to render the image. Finally, hybrid direct volumetric rendering algorithms [24], [25] which are classified as a mix between the techniques of image order algorithms and object order algorithms.

Ray-casting developed by [21], [26] is considered the first example of a direct volume rendering technique. The proposed model includes an implementation of the direct volumetric rendering method using ray-casting. It is the most advanced and state-of-the-art on the shelve method available in literature. 


\begin{tabular}{|c|c|c|}
\hline \multicolumn{3}{|c|}{ Direct volume rendering algorithms } \\
\hline Hybrid algorithms & Object order algorithms & Image order algorithms \\
\hline Slicing [24] & Splatting [22] & Ray casting [21] \\
Shear-warp [25] & Cell projection [23] & \\
Texture-based & & \\
\hline
\end{tabular}

TABLE II: Direct volumetric rendering algorithms
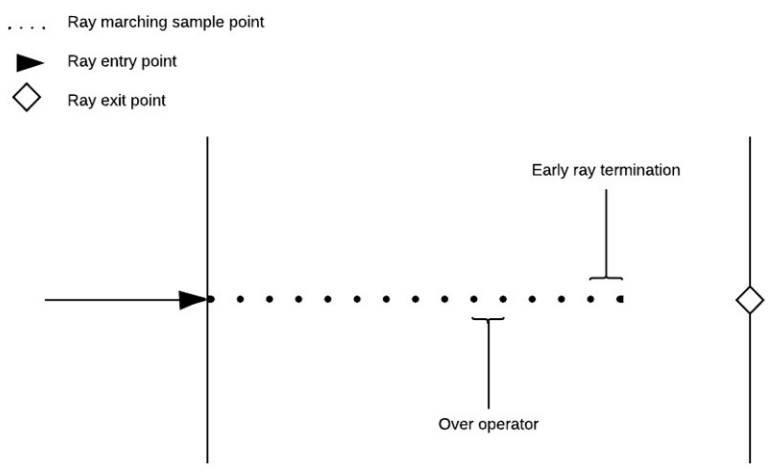

Fig. 2: Ray-march of volumetric ray-casting using an over operator with early ray termination optimisation

Volumetric ray-casting is achieved by casting rays through a volume at equally spaced sample intervals to generate the image of the 3D volume. These rays are cast through a 3D volume referred to as a 3D Voxel, the 3D point cloud containing the quadruple values. This resembles in principle to the actual acquisition of radiological data as intended by the MRI/CT scanners. When dealing with a changing angle of viewing the ray intersection may pass through a gap in data space. Furthermore, to determine the point value (colour/alpha), sample intervals are computed using interpolation. To eliminate this gap in data space, we interpolate to estimate from the surrounding known points in volume to calculate the value of the sample point intersecting the ray.

Ray-casting can be described as a front-to-back process. This is because of the order of execution as ray traverses from the axis of the camera to the back of the volume. This process of front-to-back traversal of the a single ray and its sample points is demonstrated in figure 2 . figure 3 demonstrates how

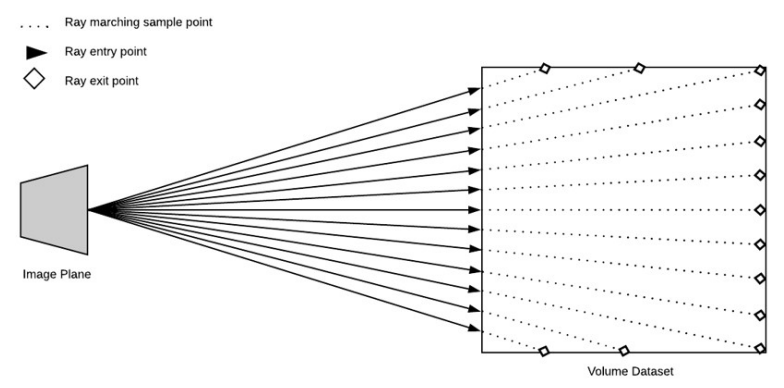

Fig. 3: A front-to-back traversal of a ray through a volume

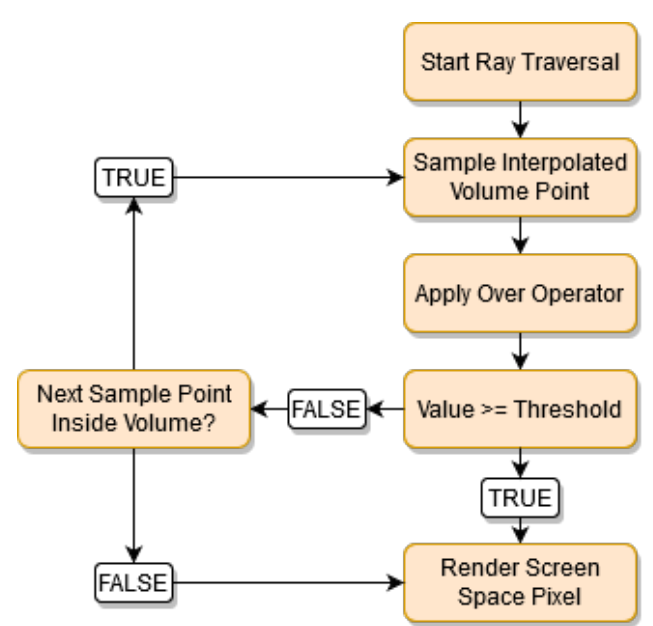

Fig. 4: Flowchart of the process of ray casting for a single image plane pixel

each propagation of these ray traversals are translated from a $3 \mathrm{D}$ volume to a $2 \mathrm{D}$ screen space. MIXR utilises a front-to-back propagation method of ray-casting this is because it allows for performance optimisations such as early ray termination. When the sample points of the rays are too dense that no further sample points can be reached, the ray can be terminated early to save computational costs. The process of early ray termination is demonstrated in figure 2 , a single ray traversal is shown through a volume. Pixels are sampled until a threshold value is reached where it is deemed that the subsequent sample points will not contribute enough to the final pixel value. Illustrated by figure 4 , the ray sample points are stopped before reaching the exit point of the volume. This avoids unnecessary processing if the subsequent data points will provide negligible contribution to the image.

To render a pixel from a 2D DICOM stack, which is intend for the screen space, into a 3D reconstructed volume in mixed reality, we perform a 'ray march' as outlined in Figure 4. As the rays are marched through the volume, we map the values to simulate the optical properties of light through composition as described by [27]. This results in an RGBA colour space corresponding to the radiative transfer of combining the RGBA of the incoming fragment (traversal sample points) with the frame buffer (destination sample points). We blend the ray marching sample points using alpha blending, alternatively known as the over operator explained in [28]. This operator can be described as:

$$
C_{o}=\frac{C_{a} \alpha_{a}+C_{b} \alpha_{b}\left(1-\alpha_{a}\right)}{\alpha_{a}+\alpha_{b}\left(1-\alpha_{a}\right)}
$$

where $C_{o}$ is the result, $C_{a}$ is the colour of pixel element $\mathrm{A}, C_{b}$ is the colour of pixel B. $\alpha_{a}, \alpha_{b}$ is the corresponding alpha of the pixel element. Assuming the colour values are scaled by the corresponding alpha values $\left(c_{i}=\alpha_{i} C_{i}\right)$, we can rewrite the output colour as:

$$
C_{o}=C_{a}+C_{b}\left(1-\alpha_{a}\right)
$$




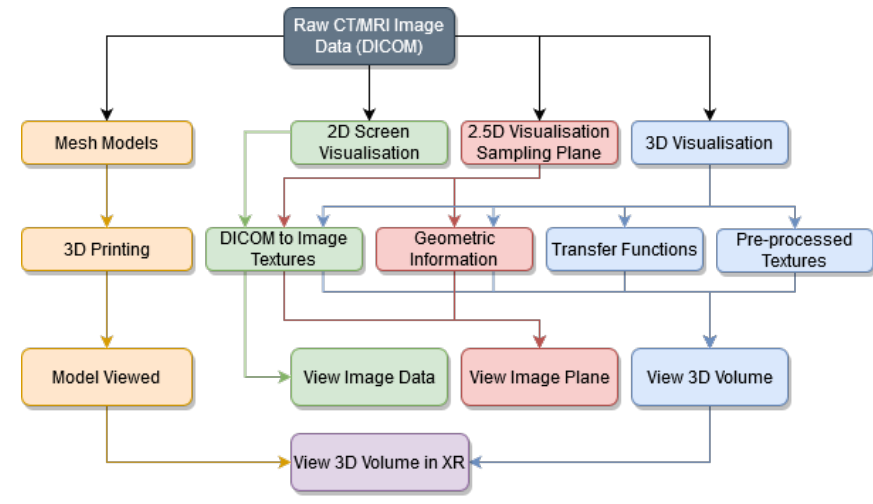

Fig. 5: Visualisation workflows for viewing radiology imagery

and the resulting alpha as:

$$
\alpha_{o}=\frac{c_{o}}{C_{o}}=\alpha_{a}+\alpha_{b}\left(1-\alpha_{a}\right)
$$

The ray-casting method is implemented through a fragment shader, are very efficient script that typically run on a graphics processing executing on every screen pixel in parallel. Shaders are simple programs that calculate the rendered screen space using vertices and pixels values (RGBA).

\section{Data Visualisation}

Visualisation of medical imaging data can take various different forms as described by [10]. The simplest application is to use raw image data shown in figure 5 to create a virtual representation on a $2 \mathrm{D}$ monitor. A variation of this work flow is to create an image plane of the volume. Referred to as a "slice", using geometric information we extract a 2D view of a slice of the $3 \mathrm{D}$ volume.

However, utilising XR systems, the orientation of a plane within the volume can be utilised to better comprehend the spatial relationship of the "slice" in the 3D volume. These systems produce a 3D rendered reconstruction of 2D imaging data with a third dimension in space, i.e. MRI or CT. It allows the user to change location and orientation of the reconstructed volume in real-time. There are a number of approaches to visualising 3D volumes but the two main workflows to achieve this is through $3 \mathrm{D}$ printing and 3D volumetric rendering.

A common technique used in surgical planning that that raw image data is reconstructed into a mesh model. This can be printed to create a physical model of the image or be visualised virtually. 3D printing has a few critical drawbacks, most notably, the properties that 3D printing has for visualisation of soft tissue. 3D printing has shown classification problems with distinguishing features [29] in specific use-cases such as breast tissue. Material differences and imaging modalities have variation in visualisation and present potential major problems for radiologists. Using 3D printing techniques in breast imaging, with mesh models it is much easier for tumours to be hidden by parenchyma in dense breasts. Direct volumetric rendering techniques unlike mesh models allow for adjustments in the compositing in real-time, allowing for a

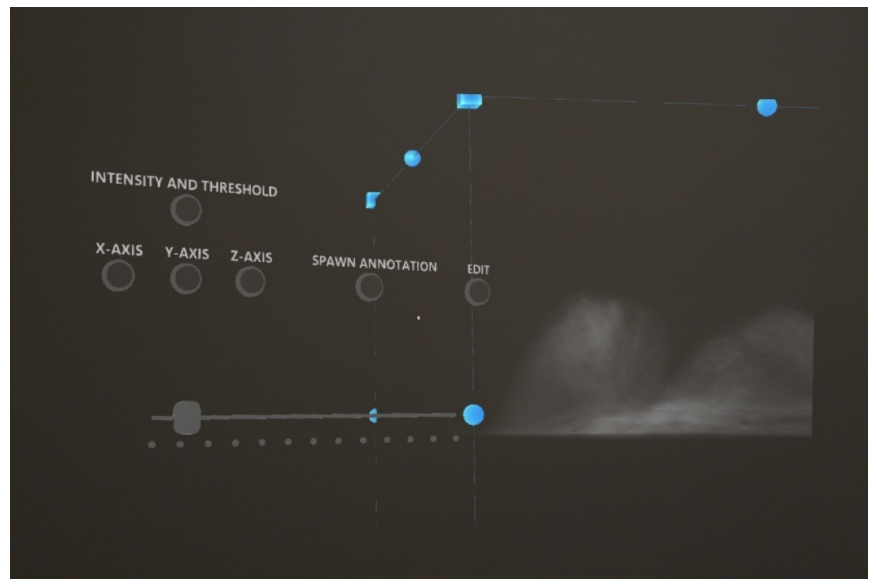

Fig. 6: 3D user interface in extended reality

far greater visualisation and isolation of regions of interest within breast CT and MRI data. Researchers [30] have found volumetric rendering has other many benefits over 3D printing in not just visualisation where users reported greater resolution but also in educational potential with XR.

\section{SySTEM OVERVIEW}

This section provides an overview of core functionalities required for a medical imaging analysis system in XR. The MIXR model has four core functionalities, that are 1) volume interaction; 2) volume exploration; 3) segmentation; 4) data annotation. These functionalities are at the heart of general clinical guidelines and norms in practice across the world.

\section{A. Volume Interaction}

Current systems used to examine radiological and oncological data implement various forms of user interfaces (UI) for $\mathrm{CT} / \mathrm{MRI}$ volume exploration. This is usually performed by the use of traditional methods relying on computer peripherals, i.e. a keyboard and mouse in conjunction with a $2 \mathrm{D}$ screen. XR does not just differentiate between a $3 \mathrm{D}$ to $2 \mathrm{D}$ visualisation, as it allows for previously unavailable ways of interaction by replacing the controller with the users hands. With this, pre-defined gestures of user's hands replace the need for a keyboard and mouse. Our XR model utilises a 3D UI shown in figure 6 which is fed and controlled by spatial motion data produced as a result of tracking the user's head and hands. Research has shown that these 3D UI can enhance user understanding of clinical data [31], thus offering an more efficient interaction system. Moreover, MIXR can be expanded for use by multiple users at the same time, where various users in the same and diverse locations can interact with the same volume at the same time. Hand gestures allow for key interactions with the volume. These includes the movement of the reconstructed volume in real space; scaling in size; rotation; colourization; zooming in and out as well as traversing through volume from all angles. The most significant feature of all is the ability to annotate a contradicted model in real space using a finger tip. 


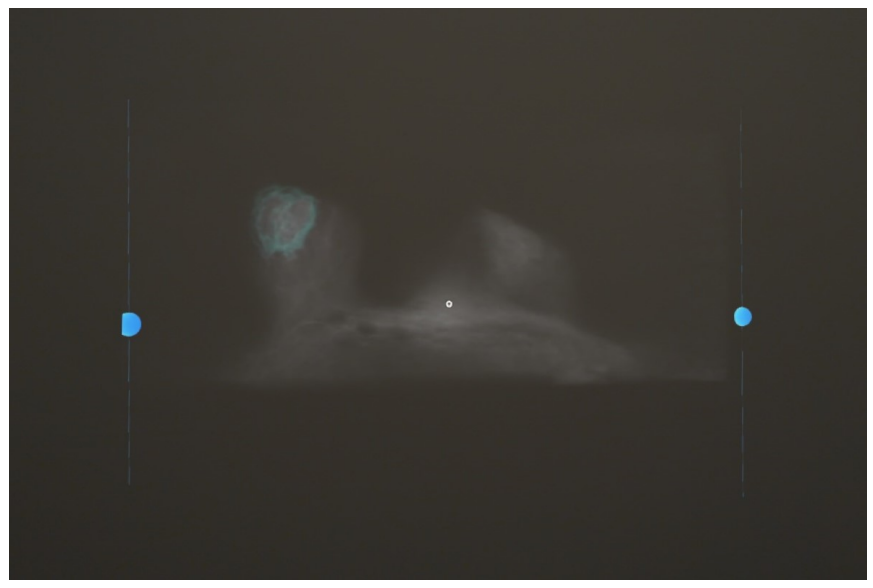

Fig. 7: User selected SLIC superpixel of breast MRI

\section{B. Volume Exploration}

There are three common methods which are used for volume exploration: 1) view aligned slicing; 2) axis-aligned slicing; and 3) user-defined slicing. These are described in further detail in [32]. To give an overview of the method that we have used in MIXR - that we consider the best aspects of all three, we use primarily a technique called axis-aligned slicing method [33], where the slicing plane is aligned to a specific axis. With the help of Microsoft mixed reality toolkit (MRTK), we have designed 3D UI shown in figure 6 for axisaligned slicing operations that would enable the radiologist to explore the volume in a $3 \mathrm{D}$ real space, however, similar to the UIs used in 2D clinical explorers. Users can control the volume using hand gestures. With the use of 3D sliders this enables the isolation of ROI's by traversing through the stack of the volume across $\mathrm{x}, \mathrm{y}$ and $\mathrm{z}$ axes. MIXR offers even further potential for more complex volume exploration techniques such as clipping planes which are user defined. To elaborate, the orientation of the hand can be used to define a clipping plane along an specified axis. This can then be used to slice along a defined plane through the volume.

\section{Segmentation}

A substantial part of the pipeline for clinical computer-aided decision, diagnostic and examination systems is outlining abnormalities. Commonly used segmentation techniques aim to isolate regions of interest (ROI's). Our model implements a few preliminary steps to bridge the gap between the medical image processing and expert interaction. The system takes expert inputs for positional data of ROI's, selected from the user's hand position when performing the pinch gesture. The positional data is related to the position within the volume and is processed to outline a segmentation boundary in 3D. We have implemented SLIC Superpixels segmentation [34] to outline ROI's within the volume, while using a seed prior from the user gesture. This provides a real-time visual of segmented region, where voxels form a 3D cluster of a volumetric region that shares similar intensity, color and spatial information (see figure 7). MIXR segments the entire parenchyma of the breast

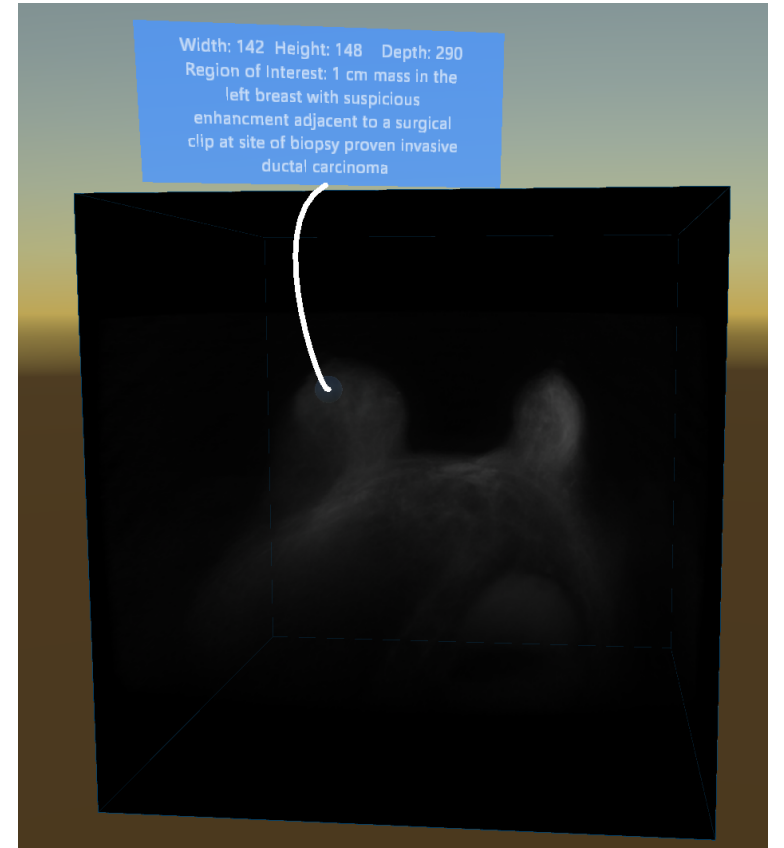

Fig. 8: Annotation of a point of interest (POI) with position and clinical notes

in $3 \mathrm{D}$, allowing the user to choose a segmented region and then further isolate the segmented region from the rest of the volume. This approach can be further refined by various pre and post processing steps, such as image enhancement and scale-space segmentation techniques, that are not discussed in this paper.

We chose SLIC Superpixels segmentation [34] for the realtime system due to its efficiency to provide fast segmentation results back to the user. However, early iterations of alternative segmentation methods deployed in XR [14] have been emerging. With a proof of concept, [14] integrated a machine learning approaches for XR. Within non-XR systems machine learning models are favoured at present as a method to perform segmentation. With our own system and these emerging XR systems now beginning to integrate current segmentation methods there is a developing need to investigate their functionality being deployed within real-time XR systems.

\section{Data Annotation}

Our model can accurately get the position of data points within the volume in $\mathrm{x}, \mathrm{y}$ and $\mathrm{z}$ axes to report an absolute point rather than relative description used in clinical reports shown in figure 8 . This point can be on the surface of the reconstructed model or deep embedded inside the stack accessed via our UI. This provide the basis for further building a framework that is able to capture, save and share clinical observations.

\section{DISCUSSION}

We propose a model that provides the basic architecture for building medical image analysis applications in XR. On 
this, we have built a fully functional mammographic image analysis system, that establishes a conceptual framework only proposed until now, into a working artefact. The algorithmic choice for volumetric rendering provides a stable 120FPS (frames per second), giving high visual clarity in XR at 720p and a consistent 60FPS when ran on the HoloLens. Running MIXR on a distributed system and remotely connecting to the HoloLens as opposed to natively running on the device ensures that a consistent frame rate is maintained whilst not impairing the real-time reactivity with minimal impact on latency. We demonstrated how the future of medical imaging in XR can move forward into radiology, oncology and radiomics by showing an application for evaluation and eventually use in breast clinics. We discussed how XR systems have shown themselves in surgical planning to provide lower cognitive burdens on users. As well as how XR has an improved cognitive experience for radiologist with improved functionality in volume exploration, spatial understanding and data annotation. Allowing user's to accurately share information in real-time with precision. The system allows for controllerless environment, a useful aspect in operations rooms for radiologists as it does not require additional hardware. It builds upon the observations we have seen from research [9], [10]. It gives validation of the applicability of XR systems being utilised within breast imaging [3], [12] as well as image analysis as a whole [12]. The system demonstrates what can be done with segmentation when joining the medical image processing and expert interaction to evolve current imaging techniques. With $\mathrm{XR}$ being poised for increase in the medical field, this XR system shows the potential in developing methods to provide real-time segmented data to radiologists. Early results using MIXR as a sample show promise however, XR still has much further potential for growth still within medical image analysis.

Research will continue to develop the system and look into diversification of functionality. Aside from the functionality discussed, there are more opportunities for development such as restructuring MIXR into a distributed framework as proposed by [14]. This would not only offer performance improvements but also practical uses for clinical practices such virtual multi-disciplinary team (MDT), a practice where several specialists would sit in a room together to assess clinical cases. Whilst, SLIC provides a good solution to MIXR's functionality, there is potential improvements that can be made in the application of machine learning.

\section{REFERENCES}

[1] L. Shi et al., "Preliminary use of HoloLens glasses in surgery of liver cancer," vol. 43, no. 5, pp. 500-504, 2018.

[2] P. Bettati, M. Chalian, J. Huang, J. D. Dormer, M. Shahedi, and B. Fei, "Augmented reality-assisted biopsy of soft tissue lesions," 2020, vol. 11315, p. 113150W.

[3] S. L. Perkins, M. A. Lin, S. Srinivasan, A. J. Wheeler, B. A. Hargreaves, and B. L. Daniel, "A mixed-reality system for breast surgical planning," 2017, pp. 269-274.

[4] A. Alfonso-Garcia et al., "Real-time augmented reality for delineation of surgical margins during neurosurgery using autofluorescence lifetime contrast," vol. 13, no. 1, p. e201900108, 2020.
[5] F. Chessa et al., "Three dimensional model of the prostate and augmented reality robot assisted radical prostatectomy: A randomized controlled study to evaluate intraoperative and pathologic outcomes," vol. 19, pp. e1774-e1775, 2020.

[6] P. Pantelidis et al., Virtual and augmented reality in medical education. 2018, pp. 77-97.

[7] A. Elmi-Terander et al., "Surgical navigation technology based on augmented reality and integrated 3D intraoperative imaging: a spine cadaveric feasibility and accuracy study," vol. 41, no. 21, p. E1303, 2016.

[8] A. Meola, F. Cutolo, M. Carbone, F. Cagnazzo, M. Ferrari, and V. Ferrari, "Augmented reality in neurosurgery: a systematic review," vol. 40, no. 4, pp. 537-548, 2017.

[9] M. G. Hanna, I. Ahmed, J. Nine, S. Prajapati, and L. Pantanowitz, "Augmented reality technology using Microsoft HoloLens in anatomic pathology," vol. 142, no. 5, pp. 638-644, 2018.

[10] J. Sutherland et al., "Applying modern virtual and augmented reality technologies to medical images and models," vol. 32 , no. 1, pp. 38-53, 2019.

[11] P. J. Gorman, A. H. Meier, C. Rawn, and T. M. Krummel, "The future of medical education is no longer blood and guts, it is bits and bytes," vol. 180 , no. 5 , pp. $353-356,2000$.

[12] D. B. Douglas et al., "Augmented reality and virtual reality: Initial successes in diagnostic radiology," 2018.

[13] V. Ferrari, G. Megali, E. Troia, A. Pietrabissa, and F. Mosca, "A 3-D mixed-reality system for stereoscopic visualization of medical dataset," vol. 56, no. 11, pp. 2627-2633, 2009.

[14] L. Trestioreanu, P. Glauner, J. A. Meira, and M. Gindt, Using Augmented Reality and Machine Learning in Radiology. Springer, 2020, pp. 89-106.

[15] F. King et al., "An immersive virtual reality environment for diagnostic imaging," vol. 1, no. 01, p. 1640003, 2016.

[16] X. Wu et al., "Mixed reality technology launches in orthopedic surgery for comprehensive preoperative management of complicated cervical fractures," vol. 25, no. 4, pp. 421-422, 2018.

[17] F. Cutolo et al., "A new head-mounted display-based augmented reality system in neurosurgical oncology: a study on phantom," vol. 22, no. 1, pp. 39-53, 2017.

[18] P.-Y. Chiang, C.-C. Chen, and C.-H. Hsia, "A touchless interaction interface for observing medical imaging," vol. 58, pp. 363-373, 2019.

[19] K. Engel, M. Hadwiger, J. M. Kniss, A. E. Lefohn, C. R. Salama, and D. Weiskopf, Real-time volume graphics. 2004, pp. 29-es.

[20] U. Technologies, Unity - Scripting API. 2020.

[21] M. Levoy, "Efficient ray tracing of volume data," vol. 9, no. 3, pp. 245-261, 1990.

[22] P. E. Undrill et al., "Integrated presentation of 3-D data derived from multisensor imagery and anatomical atlases using a parallel processing system,” 1992, vol. 1653, pp. 2-16.

[23] P. Shirley and A. Tuchman, "A polygonal approximation to direct scalar volume rendering," vol. 24, no. 5, pp. 63-70, 1990.

[24] E. Swan and R. Yagel, "Slice-based volume rendering," 1993.

[25] L. Westover, "Interactive volume rendering," 1989, pp. 9-16.

[26] M. Levoy, "A hybrid ray tracer for rendering polygon and volume data," vol. 10, no. 2, pp. 33-40, 1990.

[27] K. Engel, M. Hadwiger, J. M. Kniss, A. E. Lefohn, C. R. Salama, and D. Weiskopf, Real-time volume graphics. 2004, pp. 29-es.

[28] P. Tom Thomas and Duff, "Compositing digital images," 1984, pp. 254-259.=

[29] Y. He et al., "3D-printed breast phantom for multi-purpose and multimodality imaging," vol. 9, no. 1, p. 63, 2019.

[30] D. Bairamian, S. Liu, and B. Eftekhar, "Virtual Reality Angiogram vs 3Dimensional Printed Angiogram as an Educational tool-A Comparative Study," vol. 85, no. 2, pp. E343-E349, 2019.

[31] J. C. Goble, K. Hinckley, R. Pausch, J. W. Snell, and N. F. Kassell, "Two-handed spatial interface tools for neurosurgical planning," vol. 28, no. 7, pp. 20-26, 1995.

[32] G. Zheng, H. Liao, P. Jannin, P. Cattin, and S.-L. Lee, Medical Imaging and Augmented Reality: 7th International Conference, MIAR 2016, Bern, Switzerland, August 24-26, 2016, Proceedings, vol. 9805. Springer, 2016.

[33] B. Preim and D. Bartz, Visualization in medicine: theory, algorithms, and applications. Elsevier, 2007.

[34] R. Achanta, A. Shaji, K. Smith, A. Lucchi, P. Fua, and S. Süsstrunk, "SLIC superpixels compared to state-of-the-art superpixel methods," vol. 34, no. 11, pp. 2274-2282, 2012. 